\title{
Comportamiento Tribológico y Microestructural en Recubrimientos Aplicados por GTAW y HVOF (Proceso Térmico de Espreado) y Usado en Recuperación de Aceros Grado Herramienta AISI/SAE D2
}

Carlos Alberto Guevara Chávez ${ }^{1}$, Jorge Leobardo Acevedo Dávila ${ }^{1}$, Pedro Hernandez Gutierrez ${ }^{1}$, Jose Jorge Ruiz Mondragon ${ }^{1}$, Patricia del Carmen Zambrano-Robledo ${ }^{2}$

${ }^{1}$ Corporación Mexicana de Investigación en Materiales S.A de C.V., Technological Development Department, Saltillo, Coahuila, México.

2 Universidad Autónoma de Nuevo León, Centro de Investigación e Innovación en Ingeniería Aeronáutica, San Nicolás de Los Garza, Nuevo León, México.

Recibido: 04 Feb., 2016

Aprobado: 23 Jun., 2016

E-mails: carlosguevara@comimsa.com (CAGC), jacevedo@comimsa.com (JLAD), pedroh@comimsa.com (PHG), jjorge.ruiz@comimsa.com (JJRM), patricia.zambranor@uanl.edu.mx (PCZR)
Resumen: Las aleaciones de Fe-Cr-Mo se utilizan en recubrimientos para proteger componentes que están sometidos a condiciones de desgaste y corrosión. La alta velocidad de deposición del metalizado térmico (HVOF) (High Velocity Oxi-Fuel) aplicado en aleaciones de acero grado herramienta para trabajo en frío con la aplicación de sustratos Fe-Cr-Mo ha mostrado buenos resultados en los campos de proyección. El HOVF es un método atractivo para aplicar revestimientos y recuperar diámetros que fueron desgastados durante el proceso de operación. Nuevas superficies y materiales pueden ser provistos sin la distorsión causada por los procesos convencionales de soldadura. Para aquellos materiales que son susceptibles a agrietarse gracias a la formación de fases fuera de equilibrio duras con composiciones con altos contenidos de carbono y de cromo, debido a las condiciones del proceso que combinan una temperatura de la flama relativamente baja y con un tiempo de baja exposición. La microestructura las características del recubrimiento son determinadas por las propiedades físicas y químicas de las partículas impregnadas en el sustrato que a su vez dependen de una gran cantidad de parámetros como el diseño de pistola, la relación de oxígeno / combustible, método de inyección, tamaño de partícula y forma, entre otros La presente investigación estudia la influencia de los parámetros de procesamiento sobre las propiedades mecánicas y microestructurales del recubrimiento Diamalloy 1008 (aleación en mezcla de Fe-Cr-Mo) revestimiento aplicado con Oxí-combustible de alta velocidad (HVOF) y cinco diferentes tipos de electrodos aplicados por soldadura, TIG (GTAW) en un acero grado herramienta para trabajo en frio. El objetivo de estos recubrimientos se utiliza habitualmente como una protección contra la corrosión y el desgaste, pero también tienen la capacidad de recuperar las zonas dañadas como es el caso de los dados de estampado de la industria automotriz. Las muestras se caracterizaron mediante microscopía óptica y electrónica de barrido para evaluar la calidad del material depositado en el sustrato; microdureza Vickers y Pin-Ball Disc se utilizaron para cuantificar las propiedades mecánicas de los recubrimientos.

Palabras-clave: GTAW; HVOF; Desgaste.

\section{Tribological and Microstructural Behavior of Coatings Applied by GTAW and HVOF-thermal Spraying Process and Used in Recovering of AISI/ SAE D2 Tool Steel}

\begin{abstract}
The Fe-Cr-Mo alloys are use in covers to protect the components that are under wear and corrosion. The high velocity thermo spray covering by HVOF (high velocity oxi-fuel) in Fe-Cr-Mo alloys has reported as a good result in projection fields for the steel tool grade for cold work. The HOVF is an attractive method to apply covering and recuperate diameters. New surfaces could be provided without the distortion material caused by the weld. For those materials that are easy to crack and modified its composition due hard phases formation and high carbon content, because of the process conditions which combine a relative low flame temperature with a low exposition time. The microstructure and the covering physic properties are determined by the physical and chemical properties of the impregnated particles in the substrate that depends as well of a big amount of parameters as the pistol design, oxygen/fuel relation, injection method, particle size and shape, among others This research studies the influence of the processing parameters on the mechanical and microstructural properties of a Diamalloy 1008 (Fe-Cr-Mo alloy) coating applied with high velocity oxy-fuel (HVOF) and five different electrodes types were applied by Gas tungsten Arc Welding (GTAW) on cold
\end{abstract}


Comportamiento Tribológico y Microestructural en Recubrimientos Aplicados por GTAW y HVOF

(Proceso Térmico de Espreado) y Usado en Recuperación de Aceros Grado Herramienta AISI/SAE D2

work tool steel mold specimens. These coatings are commonly used as a protection against corrosion and wear, but they also have the capacity to recover damaged areas as toolbox for automotive stamping. The specimens were characterized by optical and scanning electron microscopy to evaluate the quality of the coatings; Vickers microhardness and Pin-Ball-Disc were used to quantify the mechanical and wear performance of the coatings.

Key-words: GTAW; HVOF; Wear.

\section{Introducción}

El proceso tecnológico de conformación de metales tiene una característica esencial la cual consiste en el alto costo de las herramientas que intervienen en el mismo. Estas generalmente están sometidas a desgaste en sus zonas de trabajo, cargas de impacto, trabajo en caliente, los esfuerzos a tensión ó compresión de su estructura debido a las configuraciones complejas de estas herramientas y, por tanto, la no uniformidad en la distribución de las cargas a que se someten, entre otros aspectos. El uso de la soldadura de reparación es una opción principal, ya que este proceso posibilita devolver a la pieza, con suficiente eficiencia y bajo costo, sus características dimensionales, propiedades mecánicas y tribológicas superiores. Las aleaciones Fe-Cr-Mo son usadas en recubrimientos para la protección de los componentes sometidos al desgaste y corrosión [1]. Los recubrimientos de rociado térmico a alta velocidad (HVOF - por sus siglas en ingles High velocity oxygen fuel) en aleaciones Fe-Cr-Mo han sido reportados con buenos resultados en el campo de las proyecciones para los aceros grado herramienta para trabajado en frio.

El HVOF es un método atractivo para aplicar soldadura de revestimiento y recuperar diámetros [2]. Nuevas superficies pueden ser provistas sin la distorsión causado por soldadura [3]. Para aquéllos materiales que son sensibles al agrietamiento y cambios en la composición debidos a la formación de fases frágiles, la formación de carburos primarios y secundarios $\mathrm{M}_{23} \mathrm{C}_{6}$ y $\mathrm{M}_{7} \mathrm{C}_{6}$. Debido a las condiciones del proceso, las cuales combinan una relativamente baja temperatura de flama con un bajo tiempo de exposición [4]. Este método emplea una alta presión de un combustible (propileno, acetileno, propano o gases de hidrógeno) para producir temperaturas del gas superior a los 3,029 @ $\mathrm{K}$ y para generar una supersónica o hipersónica velocidad del gas, de aproximadamente $2,000 \mathrm{~m} / \mathrm{s}$, más de cinco veces la velocidad del sonido [5]. El acero grado herramienta para trabajado en frío es un material susceptible al agrietamiento y formar estructuras fuera de equilibrio lo cual es un factor crítico en este tipo de componentes. La microestructura y las propiedades físicas de los recubrimientos aplicados por la técnica de HVOF están determinadas por las propiedades físicas y químicas de las partículas impregnadas en los sustratos y los materiales de aporte usados [6]. El presente estudio considera la aplicación del HVOF a componentes desgastados y compararlo con la aplicación de soldadura por proceso convencional GTAW y observar propiedades microestructurales, dureza y resistencia al desgaste, así como la calidad de la reparación entre los dos métodos propuestos.

\section{Procedimiento Experimental}

Para el desarrollo experimental se utilizaron dos procesos de soldadura, la técnica GTAW la cual se emplea en la reparación convencional de aceros grado herramienta AISI/SAE D2 (punzones, matrices, dados de conformado) y la técnica no convencional de proyección térmica de alta velocidad HVOF, la cual puede aplicar diversos revestimientos sin la afectación térmica causada en los procesos convencionales de arco, debido a que la distorsión en un parámetro fundamental en la restauración de estos aceros se optó por probar diversos materiales de aporte a fin de evaluar su comportamiento de resistencia al desgaste.

La selección de un material revestido y aporte de soldadura adecuado envuelve más que la elección de las propiedades deseadas del depósito. La consideración de este debe ser dada a las condiciones, así como la función y el servicio del entorno, en suma, a las propiedades físicas y químicas del revestimiento, aporte y sustrato. El uso de sustratos como el acero grado herramienta para trabajado en frio, fue reparado usando también un "acero de herramienta correspondiente" en polvo (Fe-Cr-Mo) para reparar materiales para el caso del HVOF y cinco tipos de aporte para el caso del GTAW, estos aportes fueron seleccionados debido a las propiedades de alta dureza y resistencia al desgaste ya que su composición química es capaz de proveer formación de carburos primarios en la matriz, por lo que los aportes seleccionados para la aplicación del proceso convencional GTAW buscan 
proporcionar diversas propiedades de superficie, esto con el fin de evaluar su comportamiento a los fenómenos de degaste (abrasión-impacto) presentes en el proceso de conformado. En la Figura 1 se muestran parte de los recubrimientos realizados por la técnica a) TIG, b) aplicación por HVOF y la infraestructura usada para su deposición, por el proceso c) HVOF. Todas las muestras usadas con la técnica HVOF tienen las mismas dimensiones totales, son bloques rectangulares de 1" pulgada $(25.4 \mathrm{~mm})$ de largo por 1/4" de pulgada $(6.35 \mathrm{~mm})$ de espesor, mientras que las usadas por la técnica GTAW en los diversos revestimientos tienen dimensiones de $4 \frac{1}{4}$ pulgadas $(108 \mathrm{~mm})$ de largo por 2 pulgadas $(50.8 \mathrm{~mm})$ de ancho por 1/4" de pulgada $(6.35 \mathrm{~mm})$ de espesor, lo cual se ilustra en la Figura 1.
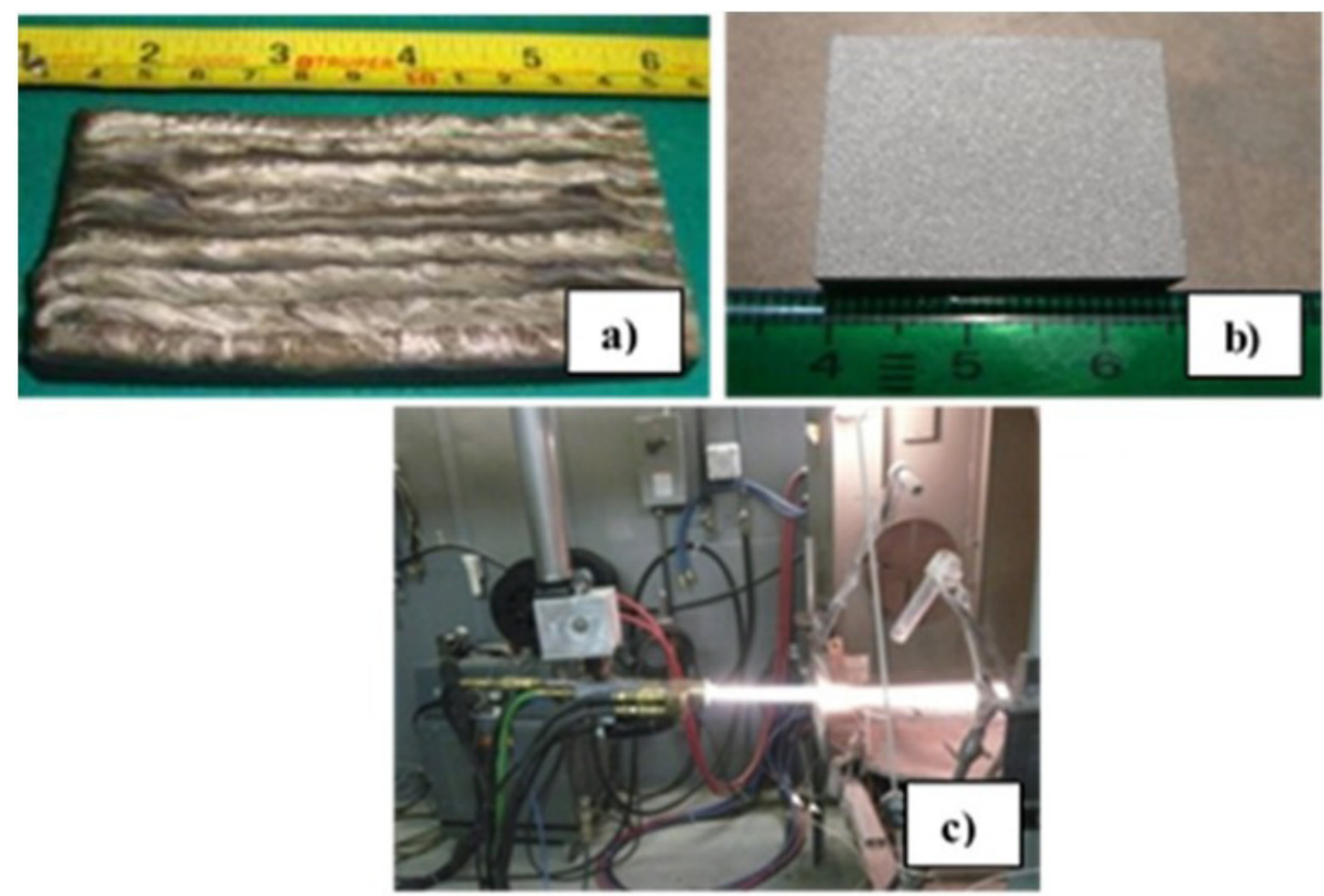

Figura 1. Recubrimiento metálico sobre substrato realizado por (a) TIG; (b) HVOF, e infraestructura empleada para deposición de recubrimientos; y (c) Equipo HVOF.

Las muestras fueron maquinadas, desgrasadas y sanblasteadas con el abrasivo de óxido de aluminio inmediatamente antes del rociado del recubrimiento, las muestras fueron precalentadas en un rango de temperatura de 250 a $350^{\circ} \mathrm{C}$ usando flama de la pistola de HVOF y un soplete de gas, esto para reducir la diferencia de expansión térmica entre la soldadura y el sus trato. Posteriormente fueron cubiertas con el material reparador y la soldadura en toda la cara superior de la muestra hasta espesores en el rango 0.5 a $1.5 \mathrm{~mm}$ para el caso del HVOF y $10 \mathrm{~mm}$ para el proceso GTAW. El proceso de HVOF fue llevado a cabo de acuerdo a los parámetros de rociado listados en la Tabla 1, para el caso de las soldaduras por GTAW se elaboró un procedimiento de soldadura enlistado en la Tabla 2.

Tabla 1. Condiciones experimentales para el proceso HVOF.

\begin{tabular}{cccc}
\hline \multicolumn{1}{c}{ Variable } & Nivel Bajo & Nivel Medio & Nivel Alto \\
Relación Propileno/oxígeno & 0,30 & 0,40 & 0,50 \\
Distancia $(\mathrm{mm})$ & 200 & 225 & 250 \\
\hline
\end{tabular}


Tabla 2. Parámetros de soldadura utilizados en la reparación del herramental por GTAW con distintos tipos de aporte.

\begin{tabular}{cc}
\hline Especificación de electrodo & AWS A5.18 \\
Diámetro del electrodo & $1 / 8^{\prime \prime}(3,2 \mathrm{~mm})$ \\
Tipo de polaridad y corriente & CDEN \\
Rango de Amperaje & $145-148$ amps \\
Tipo de electrodo & EW-Th2 \\
Rango de voltaje & $13-14$ volts \\
Velocidad de avance & Técnica 5 pulg $/ \mathrm{min}(100-127 \mathrm{~mm} / \mathrm{min})$ \\
Diámetro de boquilla de salida del gas & Oscilante \\
Tipo de gas & $1 / 2{ }^{\prime \prime}(12,7 \mathrm{~mm})$ \\
Limpieza entre pasos & Argón $99,95 \%$ \\
Precalentamiento & Cepillo y alambre \\
Postcalentamiento & 250 a $350^{\circ} \mathrm{C}$ \\
\end{tabular}

\section{Materiales y Aplicación del HVOF y GTAW}

El polvo comercial de gas atomizado Fe-Cr-Mo proveído por la empresa (Sulzer Metco Inc.,) fue usado para su estudio. La composición química y el tamaño de la partícula es mostrado en la Tabla 3. El equipo Sulzer Metco DJ 2700 Diamond Jet Gun fue usado para la aplicación del polvo, inyectando un gas propileno. Polvo fue alimentado mediante la inyección de nitrógeno con un caudal de $47 \mathrm{~g} / \mathrm{min}$. Los materiales de aporte empleados para la reparación de herramentales de conformado de acero grado herramienta por método convencional (GTAW) se utilizaron los siguientes materiales de aporte, M2, S7, Weartech, Stellite 6 y Stellite 12. Las composiciones químicas se muestran a mayor detalle en la Tabla 4.

Tabla 3. Especificación del polvo utilizado para la aplicación mediante HVOF.

\begin{tabular}{|c|c|c|c|c|c|c|c|c|c|c|}
\hline Polvo & \multicolumn{9}{|c|}{ Composición (\% en peso) } & \multirow{3}{*}{$\begin{array}{c}\text { Tamaño de la } \\
\text { partícula }(\mu \mathrm{m}) \\
\text { nominal } \\
-45+/-5,5 \mu \mathrm{m}\end{array}$} \\
\hline \multirow{2}{*}{$\begin{array}{c}\text { Fe-Cr-Mo } \\
\text { (Diamalloy 1008) }\end{array}$} & $\mathrm{Fe}$ & $\mathrm{Cr}$ & Mo & $\mathrm{Ni}$ & $\mathrm{Si}$ & $\mathrm{Cu}$ & B & $\mathrm{C}$ & $\mathrm{Mn}$ & \\
\hline & Balance & 17 & 11 & 3 & 3 & 3 & 4 & 0,4 & 0,1 & \\
\hline
\end{tabular}

Tabla 4. Composición química de los materiales de aporte utilizados en la reparación por el método de GTAW.

\begin{tabular}{|c|c|c|c|c|c|c|c|c|c|}
\hline \multirow{2}{*}{ Especificación } & \multicolumn{9}{|c|}{$\%$ en peso } \\
\hline & C & Co & $\mathrm{Cr}$ & $\mathrm{Fe}$ & Mo & $\mathbf{N i}$ & $\mathrm{Si}$ & v & w \\
\hline M2 & $0,78-1,05$ & --- & $3,75-4,50$ & Bal, & $4,50-5,50$ & 0,3 Max, & $0,20-0,45$ & $1,75-2,20$ & $5,50-6,75$ \\
\hline S7 & $0,45-0,55$ & --- & $3,00-3,50$ & Bal, & $1,30-1,80$ & --- & $0,20-1,00$ & $0,20-0,30$ & --- \\
\hline Weartech & 1,10 & Bal, & 28,00 & 3,00 Max, & --- & 3,00 Max, & 1,10 & --- & 4,00 \\
\hline Stellite 12 & $1,40-1,85$ & Bal, & 29,50 & 2,50 & --- & 3,00 Max, & 1,50 & --- & 8,50 \\
\hline Stellite 6 & 1,1 & Bal & 28,2 & --- & 1,28 & 2,1 & ---- & ---- & 5,2 \\
\hline
\end{tabular}

Un diseño de experimentos del tipo Taguchi fue usado en el estudio para evaluar el efecto de los dos parámetros (relación flujo del combustible/oxígeno, y distancia de trabajo) sobre la trayectoria de las partículas en cuanto a la velocidad y la temperatura de la misma como se observa en la Tabla 5.

Una vez recubiertas y soldadas las muestras fueron preparadas metalográficamente bajo la norma ASTM E-3. Las muestras pulidas a un acabado espejo fueron atacadas químicamente bajo la norma ASTM E-407. Con el fin de revelar la microestructura se empleó Picral y $\mathrm{HCl}$ al $4 \%$, después se realizó en ensayo de dureza en el recubrimiento, material base y en la soldadura bajo la norma ASTM E-384, con un penetrador de diamante con ángulo de $136^{\circ}$, 
Tabla 5. Diseño de experimentos tipo Taguchi.

\begin{tabular}{ccccc}
\hline $\begin{array}{c}\text { Experimentol } \\
\text { Parametro }\end{array}$ & $\begin{array}{c}\text { Flujo de } \\
\text { Combustible } \\
\text { (SLPM*) }\end{array}$ & $\begin{array}{c}\text { Flujo de Oxígeno } \\
\text { (SLPM })^{*}\end{array}$ & $\begin{array}{c}\text { Distancia } \\
\text { (mm) }\end{array}$ & $\begin{array}{c}\text { Relación Propileno / } \\
\text { Oxígeno }\end{array}$ \\
1 & 85 & 240 & 200 & 0,30 \\
2 & 85 & 240 & 225 & 0,30 \\
3 & 85 & 240 & 250 & 0,30 \\
4 & 85 & 210 & 200 & 0,40 \\
5 & 85 & 210 & 225 & 0,40 \\
6 & 85 & 210 & 250 & 0,40 \\
7 & 85 & 170 & 200 & 0,50 \\
8 & 85 & 170 & 225 & 0,50 \\
9 & 85 & 170 & 250 & 0,50 \\
10 & 85 & 240 & 200 & 0,30 \\
11 & 85 & 240 & 225 & 0,30 \\
12 & 85 & 240 & 250 & 0,30 \\
13 & 85 & 210 & 200 & 0,40 \\
14 & 85 & 210 & 225 & 0,40 \\
15 & 85 & 210 & 250 & 0,40 \\
16 & 85 & 170 & 200 & 0,50 \\
17 & 85 & 170 & 225 & 0,50 \\
18 & 85 & 170 & 250 & 0,50 \\
\hline
\end{tabular}

*litros estándar por minuto.

en escala $\mathrm{Hv}_{500}$ con una carga de 500 grf. Con equivalencia a HRC. Se realizó las pruebas de desgaste con la técnica Pin-Ball-Disc con el fin de evaluar el mejor deposito, asimismo se realizó la prueba de adherencia bajo la norma ASTM C 297/C 297M y bajo la norma ASTM D 4541. Por último, se realizó el análisis del depósito en el sustrato usando un microscopio electrónico de barrido marca Jeol con el fin de evaluar la calidad de la unión.

Para la obtención de los valores de desgaste se utilizó la técnica de Pin-Ball-Disc con equipo T-07 Abrasive wear testing machine ver Figura 2, este ensayo se realizó a las muestras descritas en la Tabla 4, los parámetros de ensayo se describen en la Tabla 6.

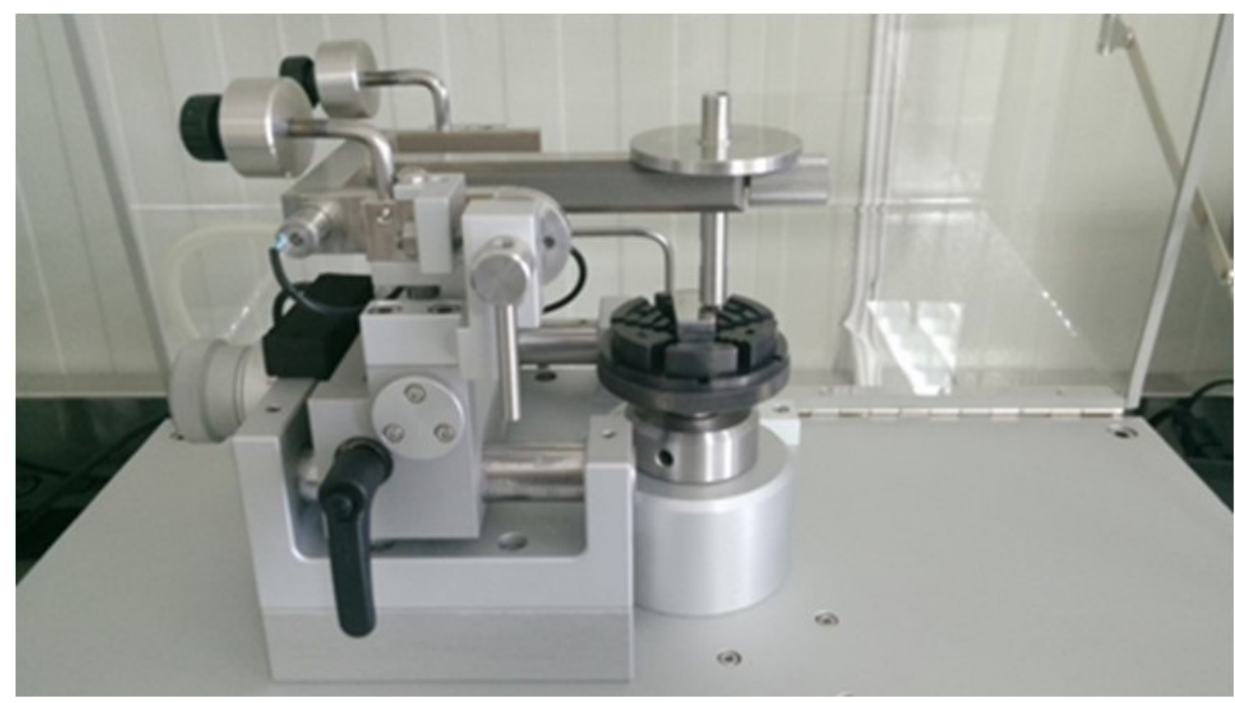

Figura 2. Aspecto general del dispositivo utilizado para el ensayo de desgaste. 
Tabla 6. Parámetros utilizados para el ensayo de desgaste fueron los siguientes.

\begin{tabular}{cccc}
\hline Parámetro & Valor & Parámetro & Valor \\
Bola de desgaste & $100 \mathrm{Cr} 6$ & Dimensión & $6,00[\mathrm{~mm}]$ \\
Geometría & Esférica & Radio & $9,50[\mathrm{~mm}]$ \\
Angulo & 45,00 & Carga & $5,00[\mathrm{~N}]$ \\
Frecuencia & $1,71[\mathrm{~Hz}]$ & Temperatura & $25^{\circ} \mathrm{C}$ \\
Ciclos & $400,0[\mathrm{ciclos}]$ & Velocidad de Adquisición & $50,0[\mathrm{hz}]$ \\
\hline
\end{tabular}

\section{Resultados y Discusiones}

Una vez aplicada la soldadura por el proceso convencional GTAW con los parámetros listados en la Tabla 2, se procedió a caracterizar las muestras (microscopia óptica, dureza, químico, desgaste), encontrándose lo siguiente EI análisis metalográfico de acuerdo a la norma ASTM E-3, E-407 revelo una microestructura compuesta por una matriz de Martensita revenida con carburos de Cromo y Molibdeno $\mathrm{M}_{23} \mathrm{C}_{7}$ y $\mathrm{M}_{7} \mathrm{C}_{6}$ dispersos en la matriz del material base, con crecimiento de grano en la dirección $\langle 100\rangle$ en la zona afectada por el calor y una matriz de granos columnares de solidificación, con precipitación interdendritica de carburos primarios en la zona de soldadura. Ver Figura 3.

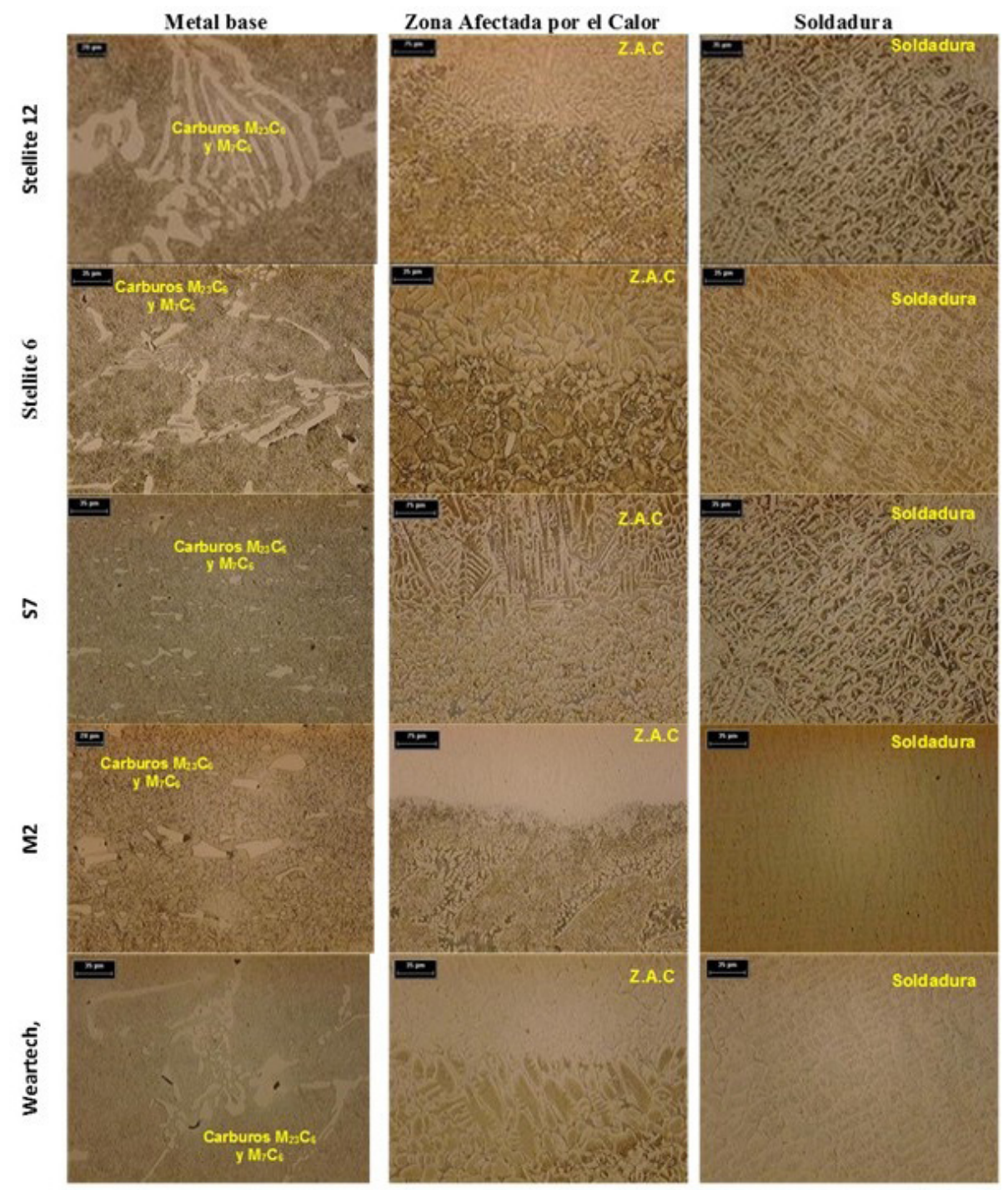

Figura 3. Martensita revenida con carburos de Cromo y Molibdeno $M_{23} C_{7}$ y $M_{7} C_{6}$ dispersos en la matriz. 
Posteriormente se realizó el ensayo de resistencia al desgaste bajo la técnica de Pin-Ball-Disc a las muestras soldadas por la técnica de GTAW con los diversos materiales de aporte descritos en la Tabla 4 y ensayados con los parámetros descritos en la Tabla 6, esto con el fin de evidenciar que material de aporte provee mejores características para la aplicación de conformado en frío, tomando el coeficiente de fricción como medida para verificar la resistencia al desgate ya que se tiene conocimiento que al incrementar el coeficiente de fricción el desgaste es mayor [7]. En la Figura 4 se muestra la variación del coeficiente de fricción con respecto al tiempo para los diferentes recubrimientos y procesos. Se observa que el recubrimiento realizado mediante HVOF es el que presenta la menor resistencia al desgaste que el TIG esto se debe a que en los procesos el tipo de anclaje difiere mientras que uno es mecánico el otro es por liga metalúrgica [5], respectivamente. De los recubrimientos depositados por TIG el que presenta mejor resistencia a la abrasión es el Stellite 12.

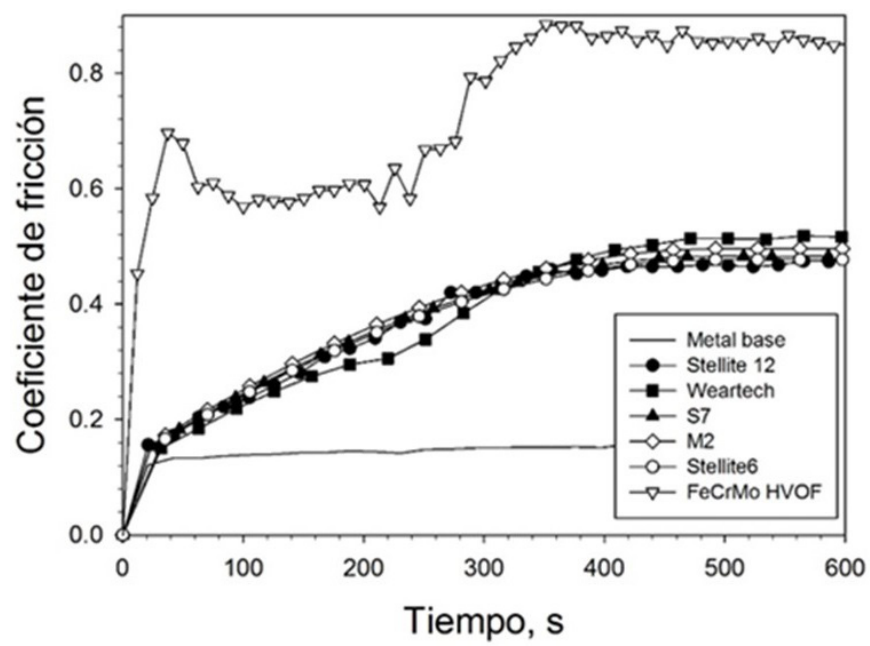

Figura 4. Resultados de resistencia al desgaste para todas las muestras estudiadas.

Una vez ajustados las condiciones de aplicación del HVOF, en la presente etapa se efectuaron 18 pruebas variando los diferentes parámetros del metalizado por proyección como se muestra en la Tabla 5 . En la Figura 5 se muestra los resultados obtenidos de los valores promedio de adherencia del diseño de experimentos aplicado para el proceso HVOF con respecto a la relación Propileno-oxígeno (c/o) y distancia, se aprecia que el valor más alto de adherencia se consigue para las relaciones de c/o más bajas y las distancias más cortas de aplicación, es decir a $200 \mathrm{~mm}$ con una relación de combustible/oxígeno de 0.30 . Aunque se observa que se pueden obtener recubrimientos con relaciones c/o mayores con adherencia similares independientemente de la distancia de aplicación en el rango examinado.

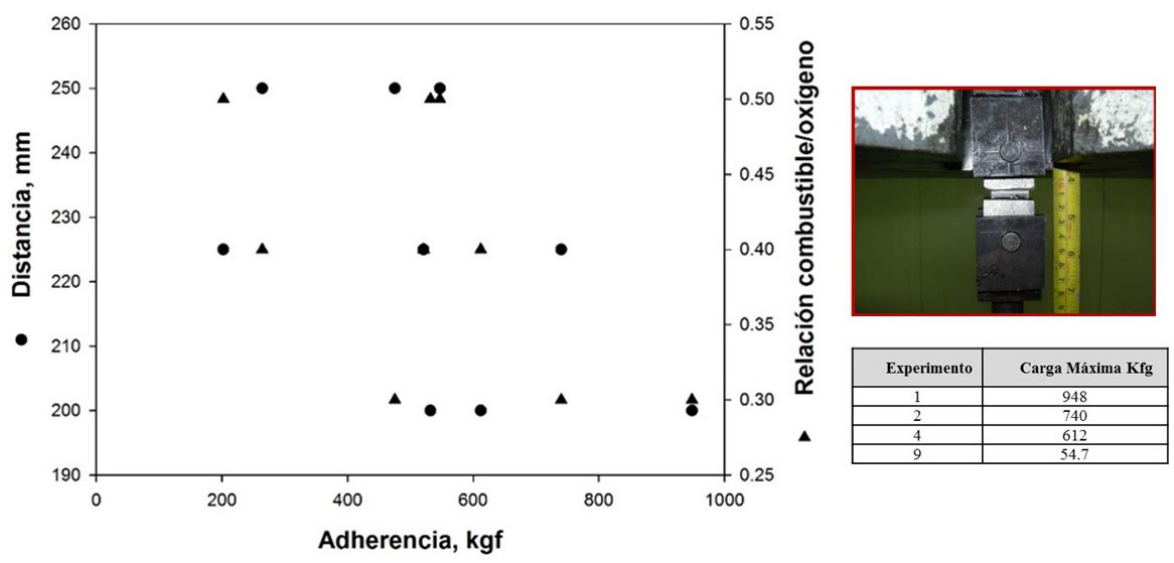

Figura 5. Resultados de adherencia del diseño de experimentos aplicado. 
En la Figura 6 se muestra la variación de la dureza con respecto al número de identación comparando dos de los recubrimientos, para diferentes relaciones de c/o manteniendo constante la distancia de trabajo. Se observa que cuando se tiene una relación c/o mayor la dureza del recubrimiento incrementa.

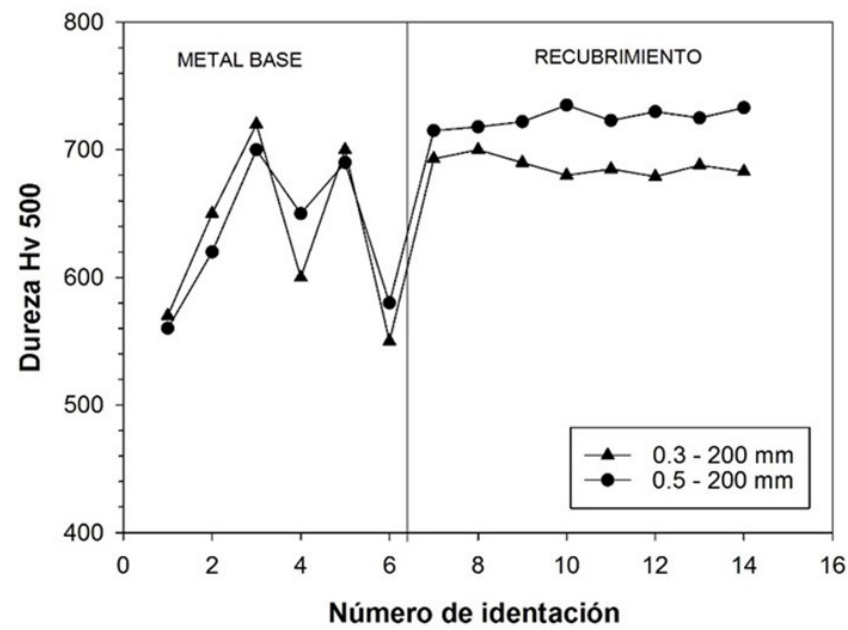

Figura 6. Relación de dureza para dos recubrimientos 0.3 c/o -200 mm y 0.5 c/o -200 mm.

En la Figura 7 se muestra imágenes obtenidas por microscopia electrónica de barrido de la zona transversal de la muestra a) 0.3 c/o - $200 \mathrm{~mm}$ y b) 0.5 c/o - $200 \mathrm{~mm}$. Se observa que la muestra a) presenta una unión mecánica en la interface sin presencia de discontinuidades sin embargo la muestra b) la muestra presenta una discontinuidad notoria en la interface lo cual perjudica de forma detrimental la adherencia del recubrimiento debido a la no coalescencia con el sustrato. Cuando las relaciones de Propileno/Oxígeno (c/o) son altas, la relación estequiometrica de combustión se aleja más debido a un mayor contenido de Oxígeno, teniendo especies oxidadas en su superficie, lo que promueve que el recubrimiento no logre un anclaje suficiente en la superficie del material que será reparado por la técnica de HVOF [8,9], lo cual afecta fuertemente su adherencia.
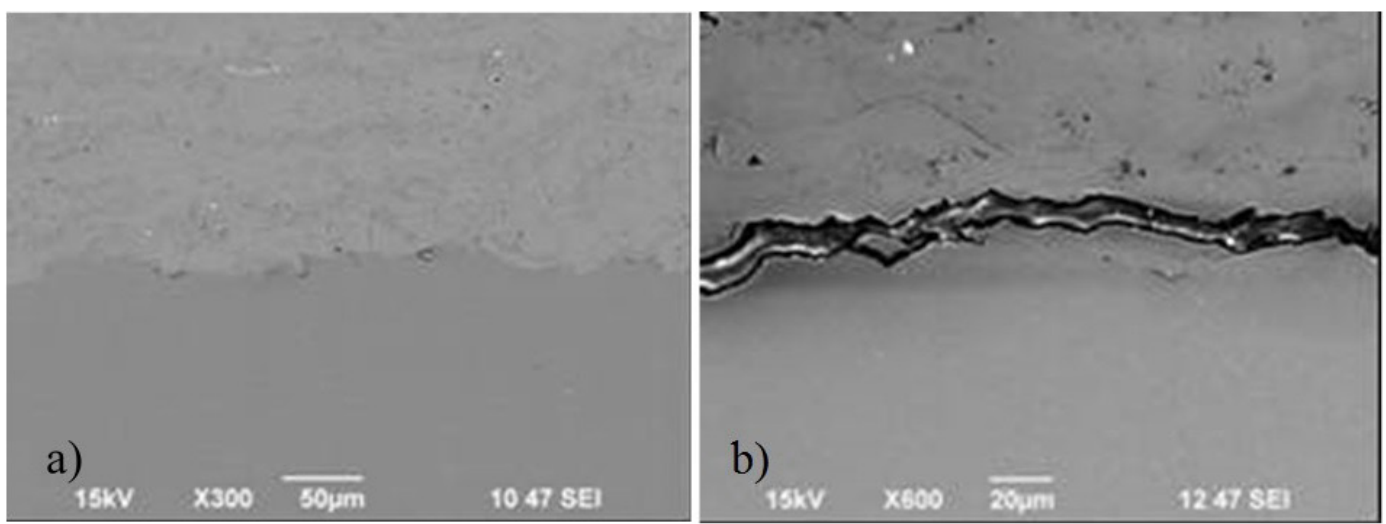

Figura 7. Imágenes obtenidas por microscopia electrónica de barrido de la zona transversal de las muestras (a) $0.3 \mathrm{c} / \mathrm{o}-200 \mathrm{~mm}$; y (b) $0.5 \mathrm{c} / \mathrm{o}-200 \mathrm{~mm}$.

\section{Conclusiones}

Las siguientes conclusiones se pueden mencionar en función a los resultados presentados:

La técnica convencional GTAW mostro resultados aceptables en cuanto a la resistencia al desgaste y propiedades similares a la del herramental, siendo el aporte Stellite 12 el de mejor compatibilidad metalúrgica con el sustrato. 
El diseño de experimentos permitió observar que para el proceso HVOF los mejores recubrimientos se obtienen al tener las distancias cortas de aplicación y relaciones de c/o bajas.

Los resultados muestran que relaciones Propileno/Oxígeno (c/o) altas promueve la formación de óxido en la superficie del material debido a la falta de combustión completa lo cual afecta directamente a la coalescencia del recubrimiento con el sustrato y promueve el desprendimiento prematuro del depósito cuando este sea sometido a tensión o desgaste.

El ensayo de dureza en el recubrimiento demuestra que el polvo seleccionado para esta aplicación cumple con las propiedades originales del herramental pudiendo así regresar el herramental a servicio.

\section{Agradecimientos}

A Comissão Editorial agradece a todos os autores e leitores deste periódico. Em particular à Associação Brasileira da Soldagem, pelo suporte financeiro a ela.

\section{Referencias}

[1] Costa JM, Ferreira JM, Capela C. Fracture toughness of the heat affected zone on Nd-YAG laser welded joints. Engineering Failure Analysis. 2008;6:93.

[2] Chavanne RL. Forty-four ways to improve the hardfacing operation. Welding Journal. 1999;62:15.

[3] Dasa D, Duttab AK, Rayc KK. Influence of varied cryo-treatment on the wear behavior of AISI D2 steel. Wear Journal. 2008;31:297-309.

[4] Borrego LP, Pires JTB. Mould steels repaired by laser welding. Engineering Failure Analysis. 2008;8:596-607

[5] Saaedi J, Coyle TW, Mirdamadi S, Arabi H, Mostaghimi J. Phase formation in a Ni-50Cr HVOF coating. Surface and Coatings Technology. 2008;202(24):5804-5811. http://dx.doi.org/10.1016/j. surfcoat.2008.05.056.
[6] Karthikeyan J, Berndt CC, Reddy S, Wong J-Y, King AH, Herman H. Nanomaterial deposts formed by DC plasma spraying of liquid feedstocks. Welding Journal. 1998;81:121.

[7] Losano G, Morgenfeld J. Criterios para evaluar el riesgo de fisuración en caliente. Revista Soldadura. 2001; 4(11):326-327.

[8] Lin YC, Wang SW, Wu KE. The wear behaviour of machine tool guideways clad with $\mathrm{W}-\mathrm{Ni}, \mathrm{W}$-Co and $\mathrm{W}$-Cu using gas tungsten arc welding. Surface and Coatings Technology. 2003;172(23):158-165. http://dx.doi.org/10.1016/S0257-8972(03)00389-X.

[9] Geng Z, Li S, Duan DL, Liu Y. Wear behaviour of WC-Co HVOF coatings at different temperatures in air and argon. Wear. 2015;330331:348-353. http://dx.doi.org/10.1016/j.wear.2015.01.035. 